\title{
Increased Fragmentation Efficiency of Ions in a Low Pressure Linear Ion Trap with an Added dc Octopole Field
}

\author{
B. A. Collings \\ MDS SCIEX, Concord, Ontario, Canada
}

\begin{abstract}
In-trap fragmentation of ions in a hybrid linear ion trap triple quadrupole mass spectrometer occurs at pressures about 5e-5 torr. At these low pressures, efficient fragmentation of heavy ions (such as the singly charged homogenously substituted triazatriphosphorine of mass $2721.89 \mathrm{Da}$ ) can take a long time because of the relatively low collision frequency with the background gas and the high internal energy content required to produce fragmentation. Increasing the amplitude used for dipolar excitation leads to loss of the ion upon the quadrupole rods. In the work presented here, the addition of a dc octopolar field to a linear ion trap is described. The dc octopolar field was created by the addition of four auxiliary electrodes situated between the quadrupole rods at a distance of $10 \mathrm{~mm}$ from the axis. The inclusion of the dc octopolar field was shown to cause the ions' frequency of motion to shift out of phase with the excitation signal at high radial amplitudes. This resulted in beat-like trajectories with periods of excitation and de-excitation as the ions' frequency of motion shifted in and out of phase with the excitation signal. This led to a reduction in the loss of ions on the quadrupole rods during the excitation process. The result is an increased fragmentation efficiency relative to the fragmentation efficiency obtained when using an LIT constructed of round rods only. The inclusion of the dc octopolar field allowed the ion to be fragmented more efficiently in a relatively short excitation period. (J Am Soc Mass Spectrom 2005, 16, 1342-1352) (c 2005 American Society for Mass Spectrometry
\end{abstract}

$\mathrm{T}$ The fragmentation of ions at pressures about $5 \mathrm{e}-5$ torr, in a low-pressure linear ion trap (LIT), requires the use of low excitation amplitudes and extended excitation periods [1, 2]. Pressures in this range are typical in the operation of hybrid linear ion trap triple quadrupole mass spectrometers that utilize the final quadrupole as both a mass resolving quadrupole and as a linear ion trap [3]. In this pressure regime, excitation amplitudes are limited from a few tens of millivolts to a few hundred of millivolts to prevent the ion from colliding with a quadrupole rod. It is the presence of the higher order field components [4-6] attributable to the use of circular rods, which enable the excitation to be carried out for extended periods of time without loss of the parent ion. A general expression for a two-dimensional multipole field can be written as

$$
\Phi(x, y)=\sum_{n=0}^{\infty} \varphi_{n}(x, y)=\sum_{n=0}^{\infty} A_{n} \operatorname{Re}\left(\frac{x+i y}{r_{0}}\right)^{n}
$$

where $2 n$ is the number of poles in a particular term. For example, $A_{2}$ is the amplitude of the quadrupole contri-

Published online June 24, 2005

Address reprint requests to Dr. B. Collings, MDS SCIEX, 71 Four Valley Drive, Concord, Ontario L4K 4V8, Canada. E-mail: bruce.collings@ sciex.com bution with the higher order terms arising from $n>2$. In the work presented here, the experimental ratio of the rod diameter to the field radius $\left(r / r_{0}\right)$ was 1.126 . It has been previously shown that the coefficients obtained from this ratio are $A_{2}=1.001462, A_{6}=0.001292$, $A_{10}=-0.002431$, and $A_{14}=-0.0002975$ with all other terms for which $n<14$ equal to zero [4]. As the ion is excited to higher radial amplitudes, the ions' motion shifts out of phase with the excitation signal attributable to the presence of terms with $n>2$. This results in the ion trajectory taking on a bead-like pattern and remaining stable within the radial trapping potential [1, 2]. For small ions, high fragmentation efficiencies approaching $100 \%$ can be achieved in less than $50 \mathrm{~ms}$ when fragmented at low $q$. Large ions may require several hundred milliseconds of excitation to achieve modest fragmentation efficiencies [1]. This is due to the increased number of collisions required to impart enough kinetic energy into the ion to cause fragmentation. Increasing the excitation amplitude results in increased loss of the ions upon the quadrupole rods. One significant drawback to using extended excitation periods is the decrease in overall scan duty cycle, an undesirable effect.

To decrease the excitation period, a number of possibilities exist. The pressure can be increased to allow for more collisions with the ion, the excitation $q$ can be increased, or higher order fields can be superposed with 
the radial trapping potential. Increasing the static pressure in a hybrid LIT triple quadrupole is not feasible because the LIT doubles as the final analyzing quadrupole and is located in the same vacuum region as the ion detector. There is an upper pressure limit in this region that exists to prevent excessive ion feedback noise and long-term damage to the detector. The excitation $q$ can be increased but with the resulting loss in mass range attributable to the low mass cut-off at $q=0.907$. Increasing the content of the higher order fields allows increased fragmentation efficiencies without sacrificing mass range. Increasing the higher order field content beyond that which is normally present within the round rod quadrupole helps to further reduce loss of the ions to the rods during the excitation process at the higher radial amplitudes. The larger the content of the higher order field the more strongly dependent the ions' frequency of motion is upon its radial amplitude [2].

Higher order fields are routinely utilized in commercial 3-D ion traps [7]. In these devices, the fields are adjusted by altering the shape or position of the electrodes, which determines the content of the higher order fields $[8,9]$. In general, the fields are adjusted to allow the ions to be ejected more rapidly from the ion trap than is otherwise possible in a 3-D quadrupole ion trap consisting of a purely quadrupolar trapping potential $[7,9]$. The result is improved mass resolution when the ions are scanned out of the 3-D trap. Franzen and Wang have devised a 3-D quadrupole ion trap in which the higher multipole field fractions can be switched on and off $[10,11]$. In this device the end cap electrodes are constructed of more than one section, allowing the content of the higher multipole fields to be varied. With the appropriate field content an ion can be fragmented more efficiently without loss of the parent ion.

In the work presented here, a set of four auxiliary electrodes were placed along the length of the LIT between the quadrupole rods at a distance of $10 \mathrm{~mm}$ from the axis of the quadrupole. The effects of the auxiliary electrodes upon the analyzing capabilities of the quadrupole, when operated in the normal mass analyzing manner, are minimized by appropriately adjusting the potentials on the auxiliary electrodes [12]. A dc potential applied to the auxiliary electrodes modified the radial trapping potential by the addition of a dc octopolar field. The addition of the dc octopolar field reduced the loss of the ions to the quadrupole rods during the excitation process, leading to an increase in fragmentation efficiencies. Through experiments and modeling, the frequency shifts and the increase in fragmentation efficiency as a function of the applied potential to the auxiliary electrodes are examined.

\section{Experimental}

\section{Experimental Set-Up}

Experiments were carried out on a modified $4000 \mathrm{Q}$ $\operatorname{TRAP}^{\circledR}$ (AB/MDS Sciex, Concord, Ontario, Canada), a

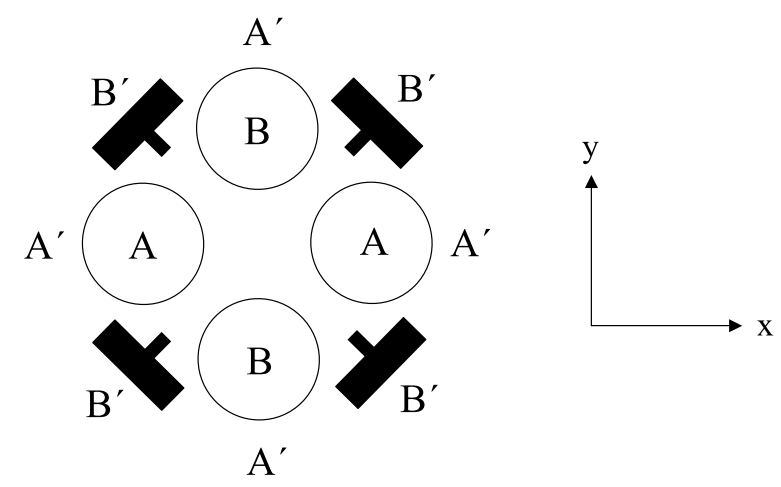

Figure 1. The electrode geometry used to create the dc octopole field. The A and $\mathrm{B}$ poles are the normal quadrupole rods. The letters $\mathrm{A}^{\prime}$ and $\mathrm{B}^{\prime}$ represent the positions of the $\mathrm{A}$ and $\mathrm{B}$ poles for the octopole. Applying a dc potential to the " $\mathrm{T}^{\text {"-shaped electrodes }}$ creates the dc octopolar field.

hybrid triple quadrupole linear ion trap mass spectrometer. The collision cell (Q2) contained typically 5 to 10 mTorr of nitrogen. The collision cell was modified to allow the axial field to be provided by a set of electrodes

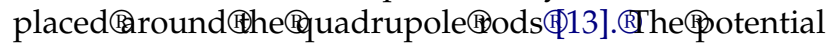
applied to these electrodes could be varied to give control over the magnitude of the axial field. The second analyzing quadrupole (Q3) performed the function of the linear ion trap (LIT) when operated in trap mode and was operated at a pressure of 3.5e-5 torr of

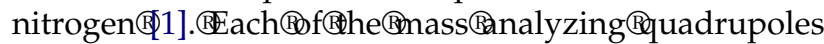
was $200 \mathrm{~mm}$ long with a field radius of $4.17 \mathrm{~mm}$. The quadrupoles were operated at a drive frequency of 816 $\mathrm{kHz}$. When operated in trap mode, the ions were scanned out of the LIT at $1000 \mathrm{Da} / \mathrm{s}$ using the technique

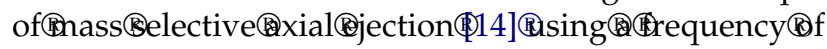
$312 \mathrm{kHz}$ for the ejection signal. Two different styles of LIT were used for the experiments described below. In one set of experiments, the Q3 quadrupole was modified by adding four $200 \mathrm{~mm}$ long auxiliary electrodes along the length of the quadrupole. In another set of experiments, the auxiliary electrodes were removed. The auxiliary electrodes each had a $2 \mathrm{~mm}$ long stem along the length of the electrode that was positioned 10 $\mathrm{mm}$ from the central axis of the quadrupole as shown in


to $+220 \mathrm{~V}$ was applied to the auxiliary electrodes relative to the Q3 quadrupole rod offset. Resonant excitation of the ions was accomplished using dipolar excitation applied between the A-pole rods of the

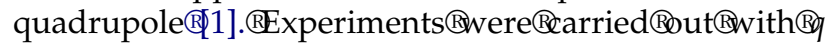
values ranging from $q \approx 0.205$ to $q \approx 0.3$. At these $q$ values, the fundamental frequency of the ions' motion ranges from 60 to $88 \mathrm{kHz}$. The amplitude and duration of the excitation ranged from 0 to $343 \mathrm{mV}$ and $100 \mathrm{~ms}$, respectively, depending upon the experiment.

The ion chosen for this study was a singly charged homogenously substituted triazatriphosphorine of

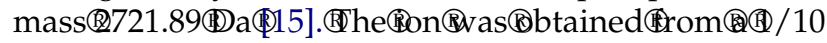
dilution of an Agilent electrospray tuning solution (Agilent ES tuning mix, G2421, Palo Alto, CA). Trap fill 




Figure 2. Fragmentation pattern observed for excitation of $\mathrm{m} / \mathrm{z}=$ $2722 \mathrm{Th}$ at $59.580 \mathrm{kHz}$. The excitation period was $100 \mathrm{~ms}$, excitation amplitude was $255 \mathrm{mV}_{0-p}$ and the excitation $q$ was $\sim 0.205$. The auxiliary electrodes were held at $+220 \mathrm{~V}$ relative to the quadrupole dc potential offset.

times were typically $50 \mathrm{~ms}$ with 25 scans co-added to produce each mass spectrum. Excitation of the isotopic cluster, at nominally $\mathrm{m} / \mathrm{z}=2722 \mathrm{Th}$, produced frag-



High-energy fragmentation spectra were collected using collision assisted dissociation (CAD). Mass spectra were collected using the EPI (enhanced product ion) mode in which the product ions are collected in the LIT before mass analysis.

Excitation of $m / z=2722$ Th at $q \approx 0.205$ and $q \approx 0.3$ results in low mass cut-offs of 615 and $900 \mathrm{Da}$, respectively. Frequency response profiles were constructed by integrating the areas of the fragment ions and the parent isotopic cluster as a function of excitation frequency. This allowed determination of the resonant frequency of the ion as a function of the applied dc potential difference between the auxiliary electrodes and the quadrupole rods.

\section{Ion Trajectory Simulations}

Ion trajectory simulations were carried out to examine two different aspects of the excitation process. The first was to determine how many collisions the ion $\mathrm{m} / \mathrm{z}=$ 2722 Th would suffer and how much center of mass collision energy was available for internal excitation during a $100 \mathrm{~ms}$ excitation period. The second aspect was to determine what effect the polarity of the auxiliary electrodes had upon the ion during the excitation process. In one set of simulations, a total of 100 ion trajectories were run using the simulator described in

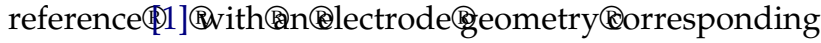
to a normal round rod analyzing quadrupole. The ions were given random starting coordinates within a $1 \mathrm{~mm}$ radius of the quadrupole axis. The ions were then cooled at a pressure of $1 \mathrm{mTorr}$ of nitrogen for a $5 \mathrm{~ms}$ period before excitation. The ions were excited using dipolar excitation with an amplitude of $80 \mathrm{mV}$ and at frequency of $59,660 \mathrm{~Hz}(q=0.205 ; 816 \mathrm{kHz}$ drive frequency). The mass of the ion was $2722 \mathrm{Da}$ and had a collision cross-section of $500 \AA^{2}$. The pressure during the simulation was $3.5 \mathrm{e}-5$ torr of nitrogen. The resonance position was found by running several ion trajectories over a range of excitation frequencies and excitation amplitudes while monitoring the duration of each trajectory. The resonance was identified by those frequencies that stopped before the maximum allowed duration, i.e., the trajectory terminated on a rod surface. The collision cross-section of this ion with nitrogen was measured using the energy loss technique described by



Modeling@f(the potential energy surfaces with various dc potentials applied to the auxiliary electrodes


simulation®program®Simion 7.0®17].®Simion $₫ 7.0 ® w a s$

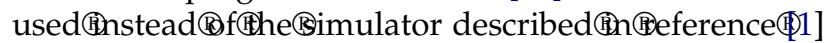
because of a limitation in the available electrode geometry of the in-house simulator at the time that 1 his $\$$ rork was undertaken. The array shown in Figure ${ }^{\circledR}{ }^{\circledR}$ was modeled@ith@n additional grounding cylinder placed at a distance of $6 \mathrm{r}_{0}$ around the quadrupole. The array was constructed using a $2000 \times 2000$ array with a resolution of 40 grid units $/ \mathrm{mm}$. This produced $r_{0}=$ $4.149 \mathrm{~mm}$ which is slightly less than the experimental $r_{0}$. To produce a more realistic $r_{0}$ would have required more RAM than was currently available on the workstation used at the time of the simulations.

Ion trajectories using $\mathrm{m} / \mathrm{z}=2722 \mathrm{Th}$ were run on this array using dc potential differences ranging from -360 to $+360 \mathrm{~V}$ between the auxiliary electrodes and the quadrupole rods. An rf amplitude of $660.9 \mathrm{~V}$ was applied to the quadrupole rods with a phase shift of 180 degrees between the A and B poles. This corresponded to $q \approx 0.205$. In one set of experiments the ions' frequency of motion was measured and plotted as a function of maximum radial amplitude attained during the simulation. In these experiments the ion was started at various radial amplitudes along the line $x=0$. The ion was given zero initial kinetic energy, no collisions were allowed and the trajectories were allowed to run for $25 \mathrm{~ms}$. Data were collected for the time and position of the ion at $1 \mu$ s intervals and then fast-Fourier-transformed to obtain the ions' fundamental frequency of motion. In another set of experiments, designed to study the effects of a positive versus negative dc potential applied to the auxiliary electrodes, the ion was started at a low radial amplitude and dipolar excitation was applied across the A pole rods. The trajectories in those experiments were allowed to run for a maximum of $25 \mathrm{~ms}$ or until the ion crashed into a rod. The ion trajectory was then examined to study the difference that the polarity of the applied potential had upon the ion trajectory.

\section{Results and Discussion}

\section{$C A D$ Experiments}

Fragmentation of the ion $\mathrm{m} / \mathrm{z}=2722 \mathrm{Da}$, using collision assisted dissociation (CAD) in the Q2 gas cell, required fairly high collision energies with very little fragmenta- 


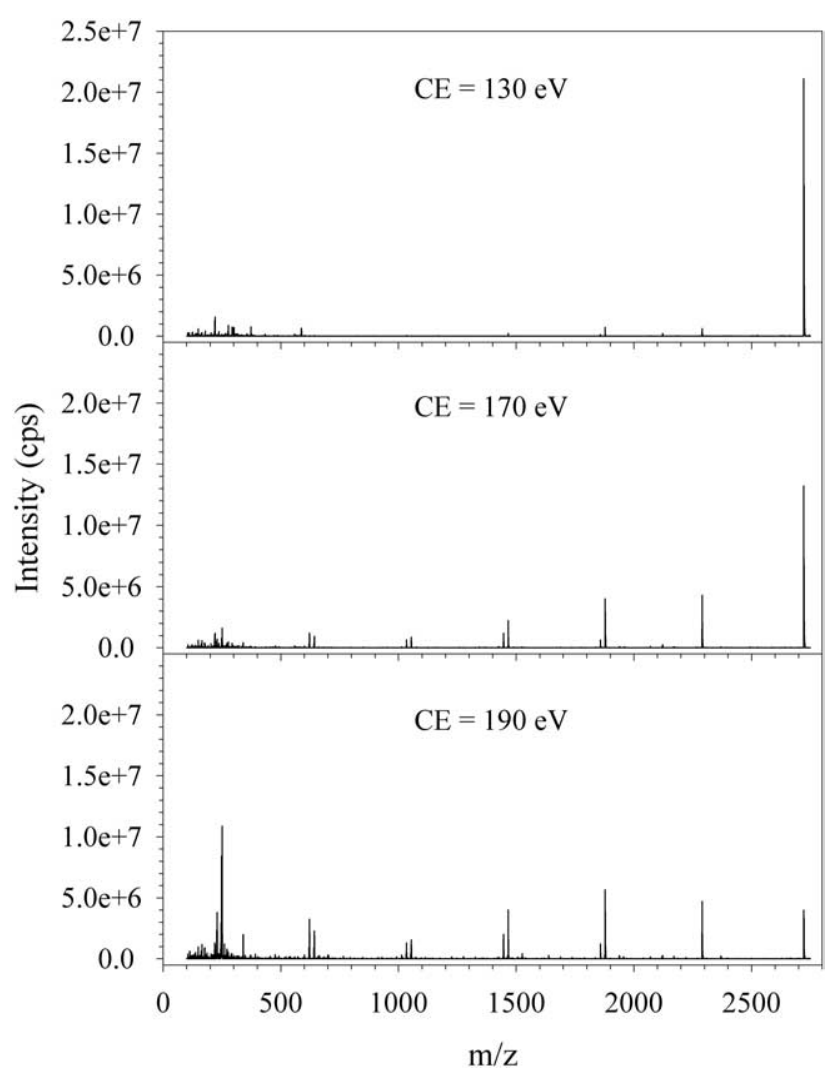

Figure 3. This figure shows the enhanced product ion spectra obtained for the ion $m / z=2722$ Th at collision energies of 130, 170, and $190 \mathrm{eV}$ with nitrogen in the Q2 collision cell. There is very little fragmentation observed at the lowest collision energy of $130 \mathrm{eV}$.

tion occurring at a collision energy of $130 \mathrm{eV}$. Mass spectra covering the mass range 100 to $2750 \mathrm{Da}$ are shown for collision energies of 130,170, and $190 \mathrm{eV}$ in

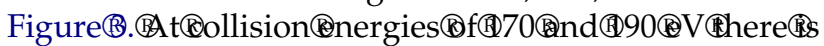
a significant amount fragmentation.

\section{Resonant Excitation Experiments}

In one set of experiments data were collected without the auxiliary electrodes in place around the quadrupole.

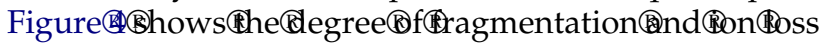
as a function of excitation amplitude obtained at $q \approx$ 0.205 and $q \approx 0.3$ for a $100 \mathrm{~ms}$ excitation period. The increase in $q$ resulted in an increase in maximum fragmentation efficiency from 10 to $35 \%$. The increase in $q$ provides a deeper pseudo-potential well that helps keep the parent and fragment ions in the LIT during the excitation process. The pseudo-potential well depth, $\bar{D}$, is@escribed(க)y

$$
\bar{D}=q \frac{V_{r f}}{4}
$$

Using $q \approx 0.205, m / z=2722 \mathrm{Da}$, and $V_{r f}=660.9 \mathrm{~V}$ gives $\bar{D}=33.9 \mathrm{~V}$. Increasing $q$ to 0.3 leads to an increase in $V_{r f}$ to $967 \mathrm{~V}$ giving $\bar{D}=72.5 \mathrm{~V}$, a significantly deeper pseudo-potential well.

An alternative method for increasing fragmentation efficiency is to modify or add higher order field components to the trapping potential. The addition of auxiliary@lectrodes, dc®octopolar field. In an octopolar field the ions frequency of motion is dependent upon its radial amplitude. As the ion is excited to higher radial amplitudes its motion shifts out of phase with the excitation frequency. The ion takes on a complicated beat-like trajectory that can be sustained for extended periods of time without the ion crashing upon a quadrupole rod. Increasing the content of the dc octopolar field results in the ion going out of phase with the excitation frequency at even lower radial amplitudes. Higher excitation amplitudes are then required to bring the ion back to high radial amplitudes. It is the high radial amplitudes that provide high kinetic energies through the ions' micromotion, giving high center-of-mass collision energies. The presence of the dc octopolar field helps to reduce the loss of ions at the high radial amplitudes. The combination of the high kinetic energy and the reduced loss at high radial amplitudes allows for an increased level of internal excitation and fragmentation

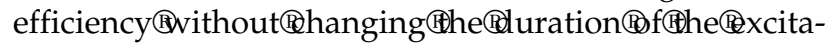

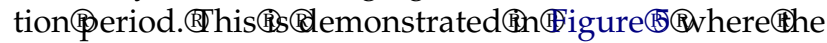
percent fragmentation efficiency is plotted as a function of excitation amplitude. In this experiment, the auxiliary electrodes were held at $+220 \mathrm{~V}$ relative to the

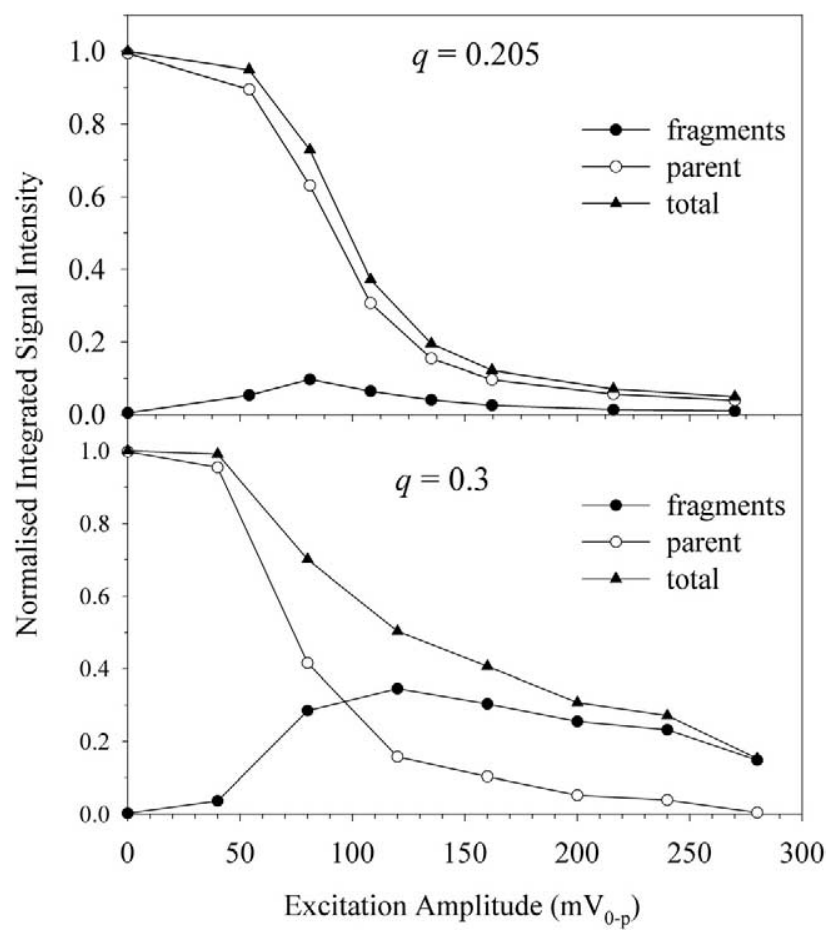

Figure 4. Normalized integrated signal intensity as a function of excitation $q$ and excitation amplitude for a LIT without the presence of the auxiliary electrodes. The excitation period was 100 $\mathrm{ms}$ in both cases. 


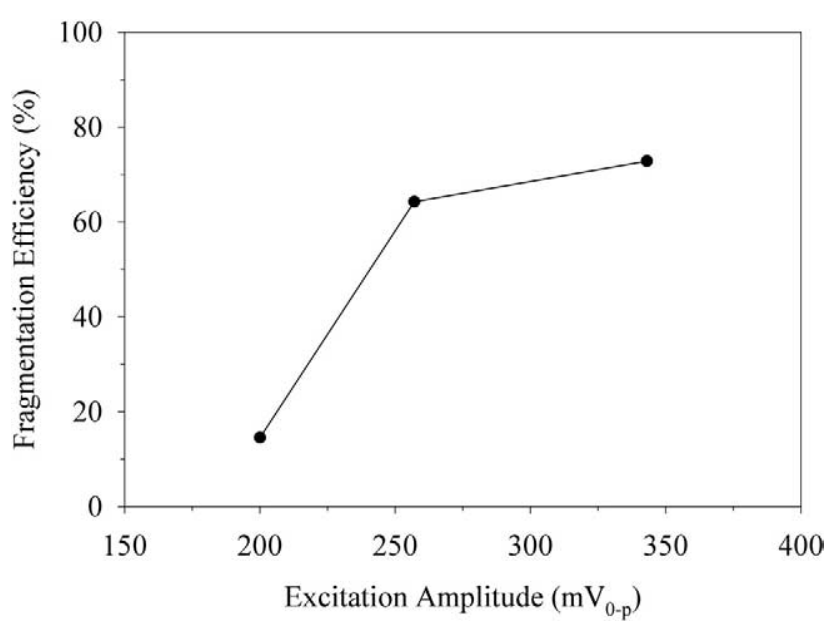

Figure 5. Percent fragmentation efficiency, at the maximum of the frequency response curve, as a function of excitation amplitude. The potential difference between the auxiliary electrodes and the quadrupole rods was constant at $+220 \mathrm{~V}$.

quadrupole rods. When the excitation amplitude was increased from 200 to $345 \mathrm{mV}_{0-p}$, the maximum of the frequency response profile shifted from 59.43 to 59.34 $\mathrm{kHz}$. The excitation period was held constant at $100 \mathrm{~ms}$.

The increases in fragmentation efficiency are also dependent upon the polarity of the dc octopolar field that is applied. It is not sufficient to simply phase-shift the ions' motion relative to the excitation signal. Experiments show that it is also necessary to apply the dc octopolar field with the same polarity as the ion of interest. Applying a negative potential difference to the auxiliary electrodes results in almost complete loss of the positively charged parent ion, whereas at the same excitation amplitude a positive potential difference leads to a high degree of fragmentation. This is shown

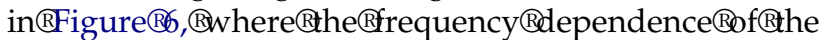
response profiles as a function of the applied potential difference to the auxiliary electrodes are plotted. The data were obtained using an excitation amplitude of 255 $\mathrm{mV}$ for each experiment. The first significant feature was a shifting of the ions' resonant frequency downwards as the potential on the auxiliary electrodes becomes more positive. The frequency decreases by $\approx 600$ $\mathrm{Hz}$ when the applied potential difference was changed from $-120 \mathrm{~V}$ to $+220 \mathrm{~V}$. With the exception of the data for a zero potential difference, the resonant frequency profiles have a FWHM of about 150 to $190 \mathrm{~Hz}$. This width is sufficient to cover several isotopes, at the nominal $\mathrm{m} / \mathrm{z}$ of $2722 \mathrm{Th}$, which are spaced by $\sim 22 \mathrm{~Hz}$ [1].医xciting(the@ntire isotope will lead to a broadening of the frequency


plot of the resonant frequency as a function of the potential applied to the auxiliary electrodes. Additional data have been included for excitation amplitudes ranging from 100 to $345 \mathrm{mV}$ when the auxiliary electrodes were present, and ranging from 40 to $110 \mathrm{mV}$ when they were absent. The data collected with no auxiliary elec-
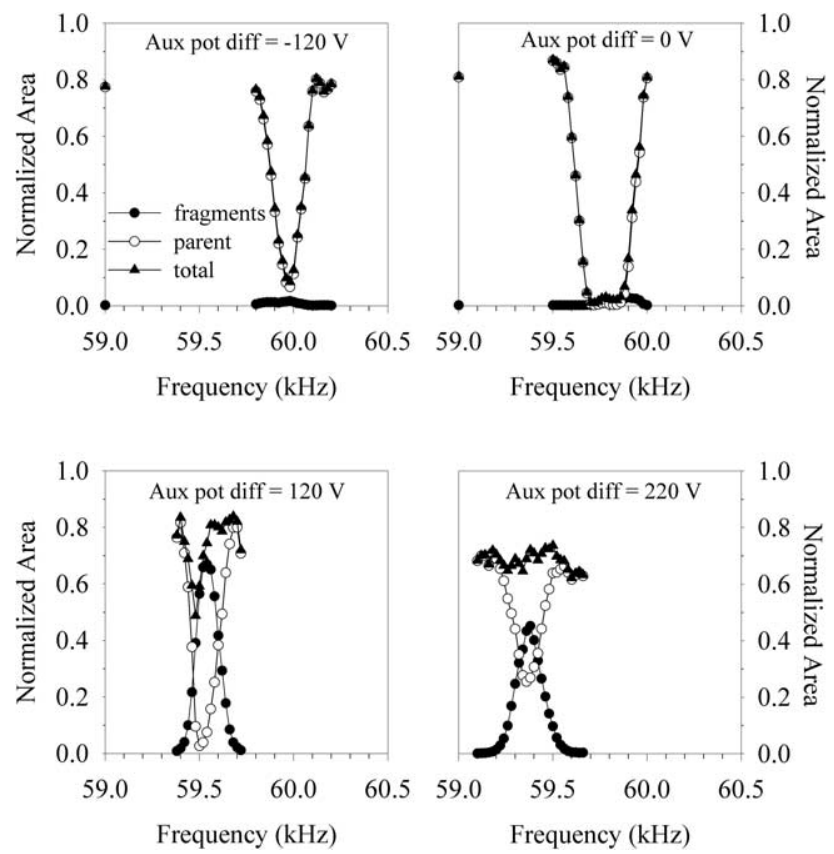

Figure 6. Frequency response profiles for $m / z=2722 \mathrm{Th}$ as a function of potential difference between the quadrupole rod dc offset and the dc potential applied to the auxiliary electrodes. The excitation amplitude of $255 \mathrm{mV}_{0-p}$ was applied for a period of 100 $\mathrm{ms}$ in each experiment. The solid circles represent the integrated intensity of the fragment ions, the open circles the parent ion cluster, and the solid triangles the total or sum of the fragment and parent ions.

trodes were obtained with lower excitation amplitudes where the resonance width was about $150 \mathrm{~Hz}$. It should be noted that the data acquired with no auxiliary electrodes is offset by $+20 \mathrm{~V}$ for clarity. The data show approximately that the resonant frequency decreased linearly as the potential applied to the auxiliary electrodes was increased.

Further investigation revealed that the resonant frequencies were dependent upon the excitation ampli-

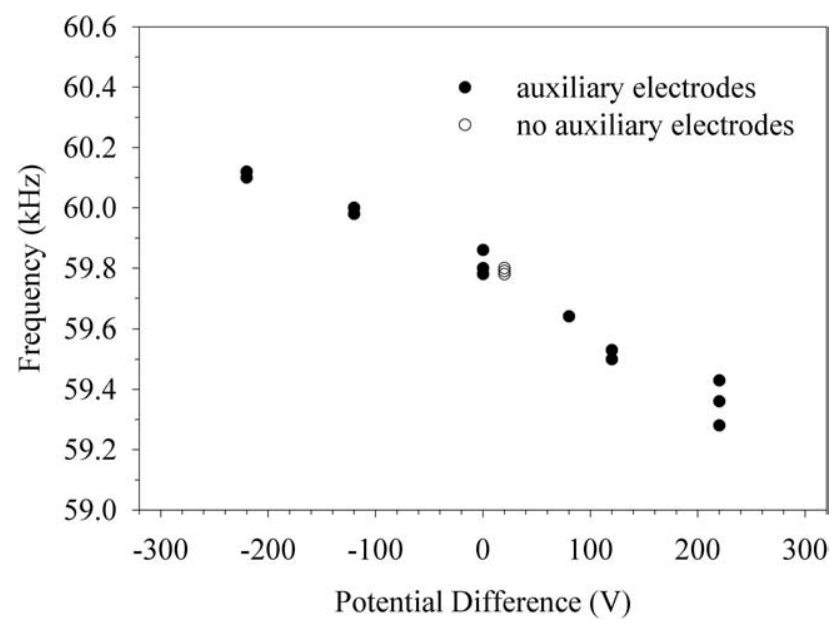

Figure 7. Plot of the resonant frequency of $m / z=2722 \mathrm{Th}$ as a function of the applied dc potential difference to the auxiliary electrodes relative to the quadrupole rod dc offset. 




Figure 8. Plot of the resonant frequency as a function of excitation amplitude. The dc potential difference applied to the auxiliary electrodes was $+220 \mathrm{~V}$ electrodes relative to the quadrupole rod dc offset.

tude. Plotting the resonant frequencies for a potential difference of $+220 \mathrm{~V}$ as a function of excitation ampli-

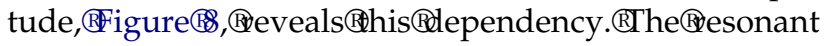
frequency shifted by $-150 \mathrm{~Hz}$ when excitation amplitude was increased from 200 to $343 \mathrm{mV}$. This can be interpreted as a decrease in the ions' secular frequency as the ion was excited to higher radial amplitudes.

The nature of the frequency shifts can be understood by examining the changes in the electric potential when a dc octopolar term is added. The electric potential can be approximated by eq 3 .

$$
\begin{aligned}
& V(x, y)=\left[A_{0}+A_{2}\left(\frac{x^{2}-y^{2}}{r_{0}^{2}}\right)\right]\left(U-V_{r f} \cos \Omega t\right) \\
& +S(x, y)\left(\frac{1}{2}-\frac{\left(x^{4}-6 x^{2} y^{2}+y^{4}\right)}{\left(r_{0}^{\prime}\right)^{4}}\right) U_{a u x}
\end{aligned}
$$

The first set of terms on the right hand side describe the electric potential due to the constant potential and the quadrupolar term. The contributions from the higher order terms normally found in a round rod quadrupole have been excluded for simplicity in eq 3 and only become significant when the second term on the right hand side is close to or equal to zero. The second term consists of the expression for an octopole, the $n=4$ term from eq 1 , with a shielding function, $\mathrm{S}(\mathrm{x}, \mathrm{y})$, applied to account for the shielding effects of the quadrupole rods. The dc octopole can be viewed as having the four rods of as the quadrupole rods. The potential applied to the $\mathrm{A}^{\prime}$ pole is then the offset potential of the quadrupole. The four@ods@f positions of the " $\mathrm{T}$ "-shaped auxiliary electrodes. Experimentally, the magnitude of the dc octopole is controlled by varying the dc potential difference between the quadrupole and the auxiliary electrodes and is the parameter $\mathrm{U}_{\mathrm{aux}}$ in eq 3 . The distance from the axis of the quadrupole to the auxiliary electrodes is $\mathrm{r}_{0}^{\prime}=10 \mathrm{~mm}$. Without the effects of shielding it is expected that the potential at the point $x, y=0,0$ should be equal to $\mathrm{U}_{\text {aux }} / 2$.

It should be noted that an expression for the shielding function $S(x, y)$ in not so easily derived. Instead, an explanation of the frequency shifts is more easily given based upon an examination of the potential using the ion trajectory simulation program Simion 7.0. The shielding effects of the quadrupole rods can be quite

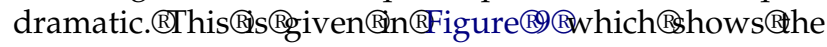
potential from the Simion model along the line $\mathrm{x}=0$

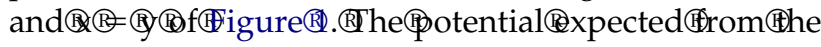
second term of eq 3 with $S(x, y)$ equal to a constant is also shown. In both cases, $V_{\text {rf }}$ and $U_{\text {aux }}$ were $0 \mathrm{~V}$ and $+220 \mathrm{~V}$, respectively. At the point $\mathrm{x}, \mathrm{y}=0,0$ the potential from the Simion model is $0.659 \mathrm{~V}$ and increases along the line $\mathrm{x}=\mathrm{y}$ towards the auxiliary electrodes. Along the line $x=0$ the potential decreases towards the quadrupole rod and becomes zero at the rod surface. In comparison, the calculated potential using a constant $\mathrm{S}(\mathrm{x}, \mathrm{y})=0.006$ produces potentials that change much less significantly and begin to noticeably deviate from the modeled potential at about $r=1.5 \mathrm{~mm}$. The value of $\mathrm{S}(\mathrm{x}, \mathrm{y})=0.006$ was derived from the knowledge of $\mathrm{V}(\mathrm{x}, \mathrm{y}), \mathrm{U}_{\mathrm{aux}}=220 \mathrm{~V}$ and eq 3 solved at the point $\mathrm{x}=0$, $\mathrm{y}=0$.

Examination of eq 3 shows that the dc octopole content will be a function of the ratio of $\mathrm{V}_{\mathrm{rf}}$ to $\mathrm{U}_{\mathrm{aux}}$. It

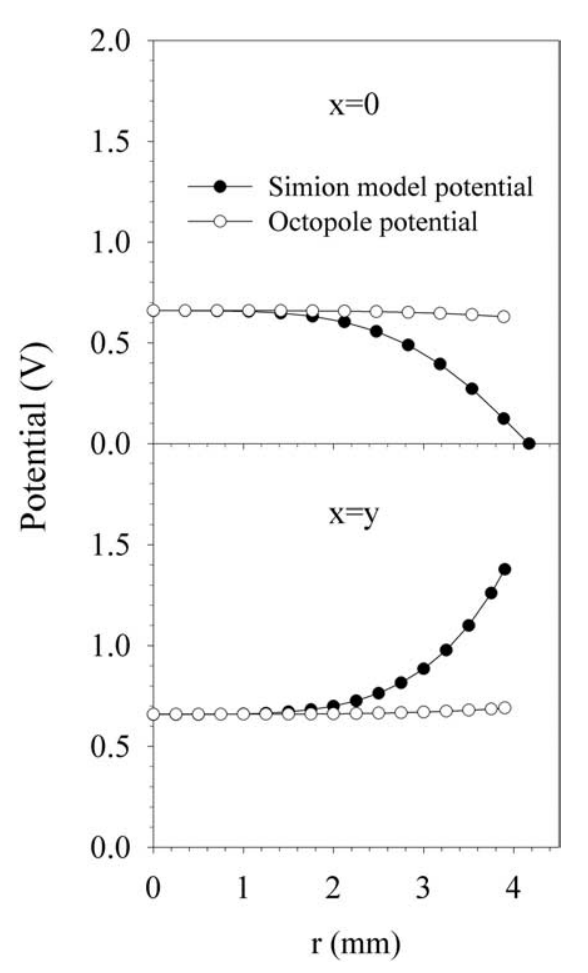

Figure 9. The potentials along the lines $x=0$, towards the quadrupole rod, and $\mathrm{x}=\mathrm{y}$, towards the auxiliary electrode for both the Simion model (solid circles) and an octopole function using a constant shielding function (open circles). 
Table 1. Frequency of ion motion as a function of $m / z, \mathrm{~V}_{\mathrm{rf}}$, and $\mathrm{U}_{\text {aux }}$.

\begin{tabular}{llrc}
\hline$m / z$ & $\mathrm{~V}_{\text {rf }}$ & $\mathrm{U}_{\text {aux }}$ & Frequency $(\mathrm{Hz})$ \\
\hline \hline 2722 & 660.9 & 0 & 60,460 \\
2722 & 660.9 & 220 & 60,270 \\
1361 & 330.45 & 0 & 60,460 \\
1361 & 330.45 & 110 & 60,270 \\
1361 & 330.45 & 220 & 60,060 \\
\hline
\end{tabular}

can be expected that the dc octopole content can be held constant with changing mass by keeping the ratio of $\mathrm{V}_{\mathrm{rf}}$ to $\mathrm{U}_{\text {aux }}$ constant. This can be shown by recognizing that in the expression for the Mathieu parameter $q$, eq 4 , the mass of the ion is proportional to $\mathrm{V}_{\mathrm{rf}}$ when $q$ is held



$$
q=\frac{4 e V_{R F}}{m r_{0}^{2} \Omega^{2}}
$$

Maintaining a constant ratio of $\mathrm{V}_{\mathrm{rf}}$ to $\mathrm{U}_{\text {aux }}$ with mass will keep the ions' fundamental frequency of motion constant. Simulations showed that decreasing $\mathrm{m} / \mathrm{z}$ from 2722 to $1361 \mathrm{Th}, \mathrm{V}_{\mathrm{rf}}$ from 660.9 to $330.45 \mathrm{~V}$, and $\mathrm{U}_{\text {aux }}$ from 220 to $110 \mathrm{~V}$ gave ion trajectories producing identical fundamental frequencies of motion; this is

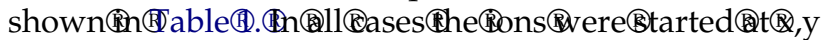
$=1.06 \mathrm{~mm}, 0 \mathrm{~mm}$ with zero kinetic energy, no collisions and no excitation. Leaving $U_{\text {aux }}$ at $220 \mathrm{~V}$ for both $\mathrm{m} / \mathrm{z}=$ $1361 \mathrm{Th}$ and $m / z=2722$ Th results in a shift of $-210 \mathrm{~Hz}$. A practical implementation of the dc octopole would require that either $U_{a u x}$ is scanned with mass while the excitation frequency is held constant or that $U_{\text {aux }}$ is held constant and the excitation frequency is scanned with mass.

\section{Simulation Results}

\section{Collision Frequency and Available Center-of-Mass Collision Energy}

Estimates of both the collision frequency and center-ofmass collision energy available for internal excitation of the ion were obtained for the round rod quadrupole LIT without the auxiliary electrodes using the ion trajectory simulator described in reference 1. A total of 100 ion trajectories were run. An excitation amplitude and frequency of $80 \mathrm{mV}$ and 59,660 Hz, respectively, were employed and the trajectories were allowed to run for up to a maximum duration of $100 \mathrm{~ms}$. A pressure of $3.5 \mathrm{e}-5$ torr of nitrogen was used in the simulation. Out of 100 trajectories 81 reached the maximum duration allowed, those that did not resulted in trajectories that terminated upon a quadrupole rod. It was found that a collision occurred on average about once every $233 \mu \mathrm{s}$ (a collision frequency of $4.3 \mathrm{kHz}$ ) with an average energy loss of $0.167 \mathrm{eV}$ per collision or a total energy loss of $72 \mathrm{eV}$ in 430 collisions in a $100 \mathrm{~ms}$ period. This compares to an average of 274 collisions and a total energy loss of $0.22 \mathrm{eV}$ in $100 \mathrm{~ms}$ when no was excitation applied. The total energy loss is equivalent to about twice the center-of-mass collision energy. The center-ofmass collision energy is equal to the maximum amount of energy that could be transferred to internal energy of the 1 ion [1].

\section{Frequency Shift versus Auxiliary Electrode Potential}

Ion trajectories were also run on the 2-D array@hown $\$$ in Figure® using®he®on®rajectory@rogram®simion 7.0. The®on® $n / z=$ \&2722® $\mathrm{R}$ was started at different radial amplitudes along the line $x=0$. The ion was given zero kinetic energy and no collisions were allowed. This produced motion along the line $x=0$ only. Data were recorded for the position of the ion every microsecond for a total of $25 \mathrm{~ms}$. The trajectory was then fast-Fouriertransformed to obtain the fundamental frequency of the

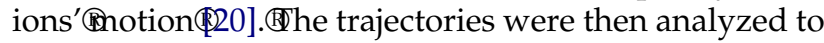
obtain the maximum radial amplitude that the ion reached during the course of the simulation. The fundamental frequency was plotted as a function of the maximum radial amplitude attained. This was carried out for a range of potentials®pplied®o®he®uxiliary

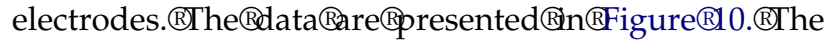
data $\$$ hows $₫$ hat when a positive potential was applied to the auxiliary electrodes, the frequency shifted downwards. When a negative potential was applied, an upward shift in frequency was observed. When the potential difference was zero, a negative shift in frequency was observed at higher radial amplitude but the magnitude of this shift was significantly less than when the potential was non-zero, particularly when $r_{\max }$ was

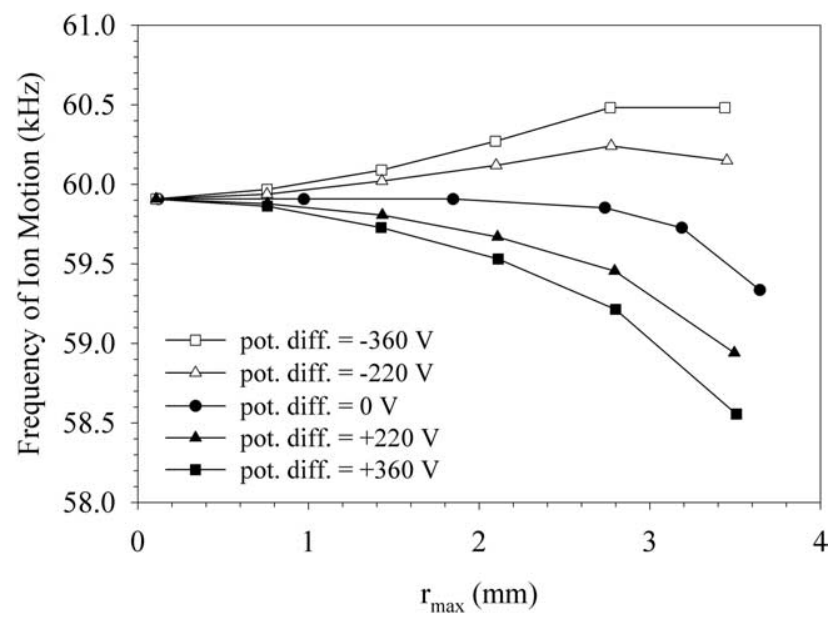

Figure 10. Frequency of ion motion along the $x=0$ vector of the

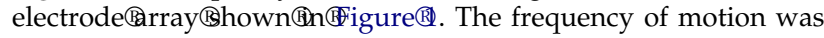
obtained by fast Fourier transforming the $25 \mathrm{~ms}$ ion trajectory used in the simulation. The data were plotted against the dc potential difference applied to the auxiliary electrodes and the maximum radial amplitude that the ion sampled during the trajectory. 


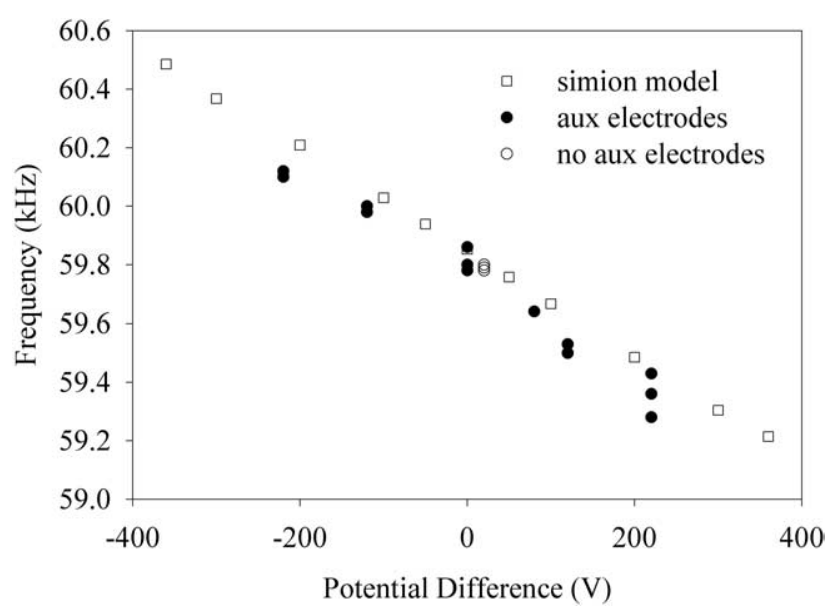

Figure 11. Experimental (solid and open circles) resonant frequencies and the resonant frequencies derived from simulations (open squares) as a function of dc potential difference between the auxiliary electrodes and the quadrupole rod offset. The frequencies derived from the simulations are from ion trajectories whose maximum radial amplitude was $\approx 2.8 \mathrm{~mm}$. The open circles represent data obtained from a quadrupole without any auxiliary electrodes.

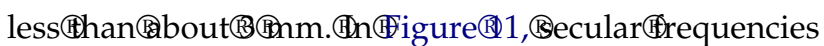
have been plotted as a function of the potential on the

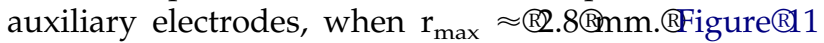
shows the same trend as the experimental data. It should be noted that the fundamental frequencies obtained from the model have been corrected for the difference in $r_{0}$ between the model and the real quadrupole. At low $q$ values $(<0.4)$ the fundamental angular frequency $w_{0}$ in a quadrupolar field can be estimated usingథ1],

$$
\omega_{0}=\beta \frac{\Omega}{2} \approx q \frac{\Omega}{\sqrt{8}}=\frac{4 e V}{m \Omega^{2} r_{0}^{2}} \frac{\Omega}{\sqrt{8}}
$$

The fundamental frequency is inversely proportional to the square of $r_{0}$. This leads to a correction factor of $0.989953\left(r_{0}(\text { model })^{2} / r_{0}(\text { real })^{2}=4.149^{2} / 4.17^{2}\right)$. The frequencies@btained this factor.

The correlation of the simulation data with the experimental data is quite good. Had the simulation data been obtained for a lower value of $r_{\max }$, the frequency shifts would have been reduced. The similarity@f(דigures $(8)$ ( radial amplitudes about $2.8 \mathrm{~mm}$ experimentally.

The direction of the frequency shifts can be understood by examining the potentials along the lines $x=0$

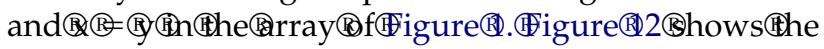
pseudo-potential well for mass $2722 \mathrm{Da}$, and the effects of the potentials from the auxiliary electrodes including the shielding by the quadrupole rods. The potentials of

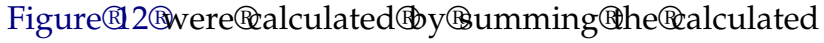
pseudo-potential with the measured static potential from the Simion model.
The expression for the pseudo-potential as a function of $\mathrm{x}$ and $\mathrm{y}$ is given by

$$
V_{e f f}(x, y)=\frac{q V_{v f}}{4}\left(\frac{x^{2}+y^{2}}{r_{0}^{2 \circledast}}\right)
$$

where $q=0.205, V_{r f}=$ (60.9 $\mathrm{Eq}{ }^{\circledR}$ is for a pure quadrupolar field with no contributions from higher order fields. Calculation of the effective potential including the non-zero contributions from the higher order terms changes the effective potential slightly at high radial amplitudes. Omission of the higher order terms does not affect the following qualitative discussion on the effects of the dc octopolar field on the ions' secular frequency.

The static potential was extracted from the Simion model with the potentials on the quadrupole rods set to $0 \mathrm{~V}$. This was done for both +220 and $-220 \mathrm{~V}$ applied to the auxiliary electrodes. The sum of the pseudopotential and the static potential produced an expected

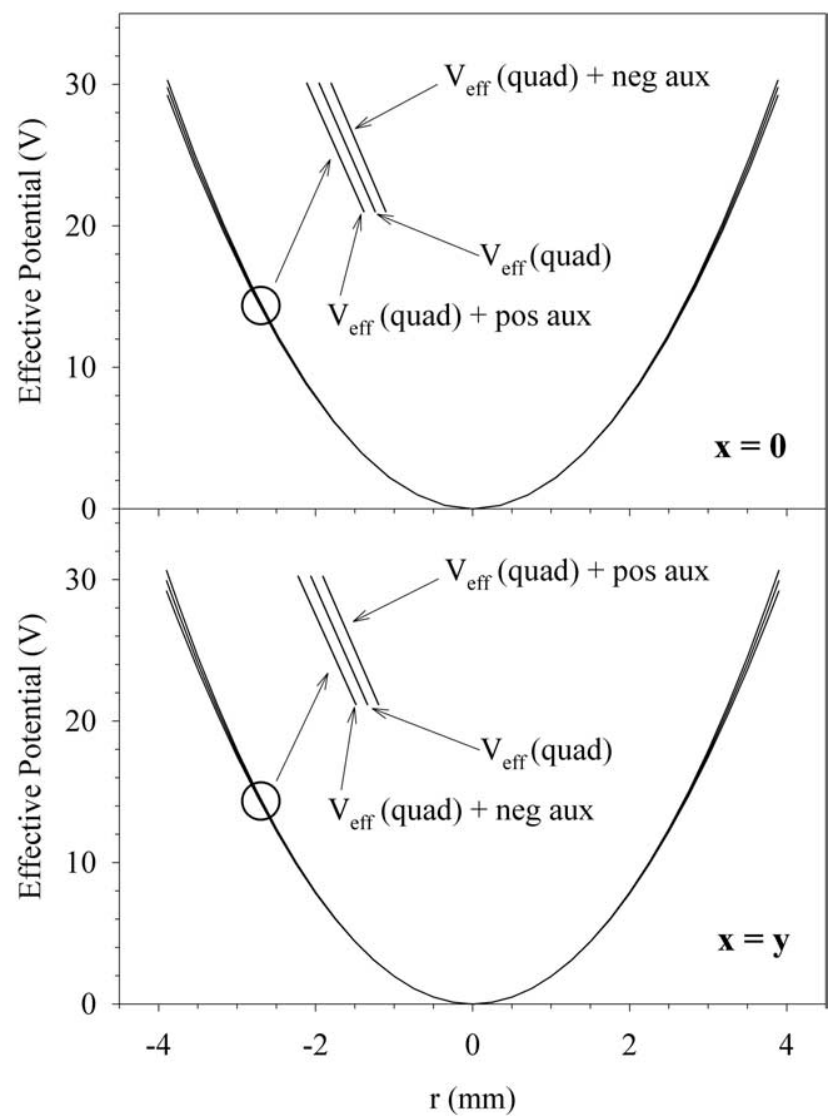

Figure 12. This figure shows the shape and depth of the pseudo potential well and the effects on the pseudo potential when a positive or negative potential is applied to the auxiliary electrodes. Applying a negative potential produces a narrower or deeper well

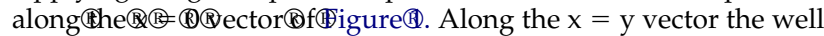
becomes shallower when a negative potential is applied to the auxiliary electrodes. The opposite effect is observed when the applied potential is positive, a deepening of the well along the $\mathrm{x}=$ $\mathrm{y}$ vector and a shallower well along the $\mathrm{x}=0$ vector. 


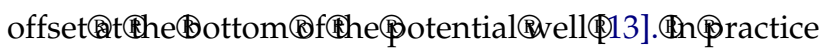
the ions are cooled to the bottom of the potential well before excitation and are unaffected by the offset. The secular frequency is dependent upon the depth and shape@fithe 12, , that bottoms of the potential wells coincide at zero. The top@rame@f line $\mathrm{x}=0$, the orientation in which the dipolar excitation is applied. A negative potential, applied to the auxiliary electrodes, causes the potential wall to rise more quickly, resulting in a deeper potential well for the same value of $r$. The opposite effect occurs when the potential applied to the auxiliary electrodes is positive: the potential well becomes shallower. The deeper well leads to an increase in the ions frequency of motion (negative potential) while the shallower well leads to a decrease in frequency (positive potential). This effect can be attributed to the shielding that the rods provide from the potentials applied to the auxiliary electrodes. The degree of shielding increases as ions approach the rods along the line $x=0$.

Along the line $x=y$, the opposite effect occurs. Applying a negative potential to the auxiliary electrodes results in a decrease in the potential well depth, while applying a positive potential results in an increase in the depth of the potential well, as shown in the bottom 4 rame@

\section{Ion Trajectory Stability}

The lack of fragmentation when a negative potential is

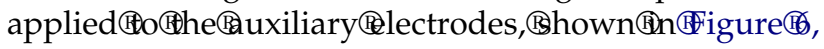
compared with the high degree of fragmentation when the potential is positive, can be understood by examining the ion trajectory during the excitation process.


potentials of 220,0 , and $-220 \mathrm{~V}$ applied to the auxiliary electrodes. The ions' motion is separated into motion parallel to the direction of excitation, $\mathrm{x}=0$, and perpendicular to the direction of excitation. In each case, the excitation amplitude was the same as the experimental value of $255 \mathrm{mV}$. The ions were excited using the frequencies $60,100,60,320$, and $60,800 \mathrm{~Hz}$ for the potentials of 220,0 , and $-220 \mathrm{~V}$, respectively. The initial starting coordinate for the trajectories shown in

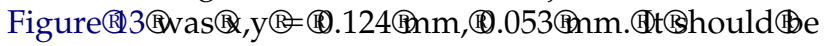
noted that the frequencies are those inputted into the Simion model and are not corrected for the difference between the values of $r_{0}$ in the model and the real quadrupole. In the case of the positive potential, the ion trajectory continued until the time limit of $25 \mathrm{~ms}$ was reached. The trajectory took on a bead-like pattern with high radial amplitude in the direction parallel to the excitation. The radial amplitude that the ion attained perpendicular to the direction of excitation remained low. The ion trajectory obtained with a zero potential applied to the auxiliary electrodes showed a linear increase in radial amplitude in the direction of excita-
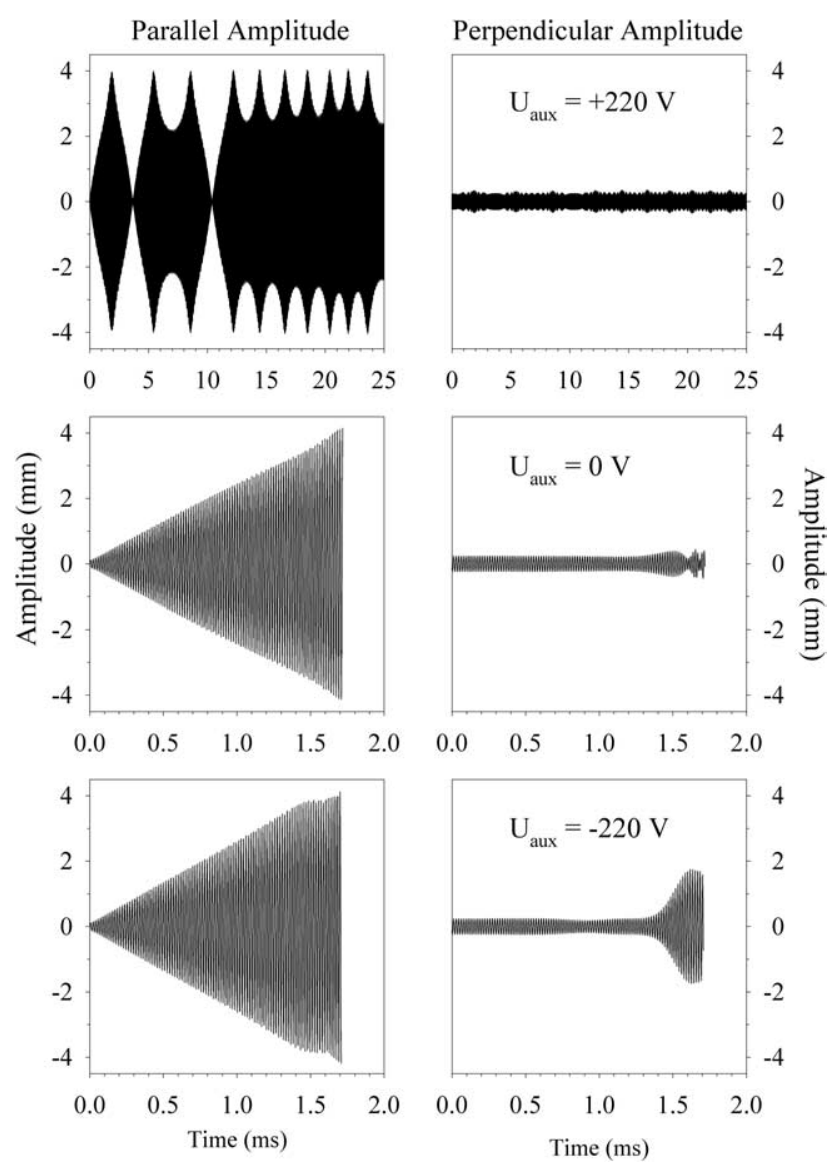

Figure 13. Representative ion trajectories are shown for when the potential applied to the auxiliary electrodes is positive $\left(\mathrm{U}_{\text {aux }}=\right.$ $+220 \mathrm{~V})$, zero $\left(\mathrm{U}_{\text {aux }}=0 \mathrm{~V}\right)$, and negative $\left(\mathrm{U}_{\text {aux }}=-220 \mathrm{~V}\right)$. An excitation amplitude of $255 \mathrm{mV}$ was used in the simulations. The left hand frames show the amplitude of motion parallel to the excitation vector $(x=0)$, the right hand frames show the amplitude of motion perpendicular to the direction of excitation. With $\mathrm{U}_{\text {aux }}=+220 \mathrm{~V}$ the ion trajectory was terminated when the time limit of $25 \mathrm{~ms}$ was reached. With $\mathrm{U}_{\text {aux }}=0 \mathrm{~V}$ the amplitude of the ion trajectory grew linearly until it terminated upon a rod at $\approx 1.7$ ms. With $U_{\text {aux }}=-220 \mathrm{~V}$ the ion trajectory terminated after $\approx 1.7$ ms when the amplitude of motion perpendicular to the direction of excitation became large and the trajectory terminated upon a rod.

tion until the trajectory terminated upon a rod surface. This occurred on a relatively short time scale, $\approx 1.7 \mathrm{~ms}$. Examination of the ion trajectory with a negative potential applied to the auxiliary electrodes showed that the ions' radial amplitude increased linearly until the radial amplitude approached $4 \mathrm{~mm}$. At this point the dc octopolar field begins to cause the ions' motion to shift out of phase with the excitation signal and coupling into motion perpendicular to the direction of excitation becomes evident. An increase in the ions' amplitude perpendicular to the direction of excitation preceded the termination of the trajectory upon a quadrupole rod. This occurred on a relatively short time scale, $\approx 1.7 \mathrm{~ms}$. The reason for the gain in amplitude, perpendicular to the excitation vector, can be understood by examining how the static potential provided by the auxiliary 

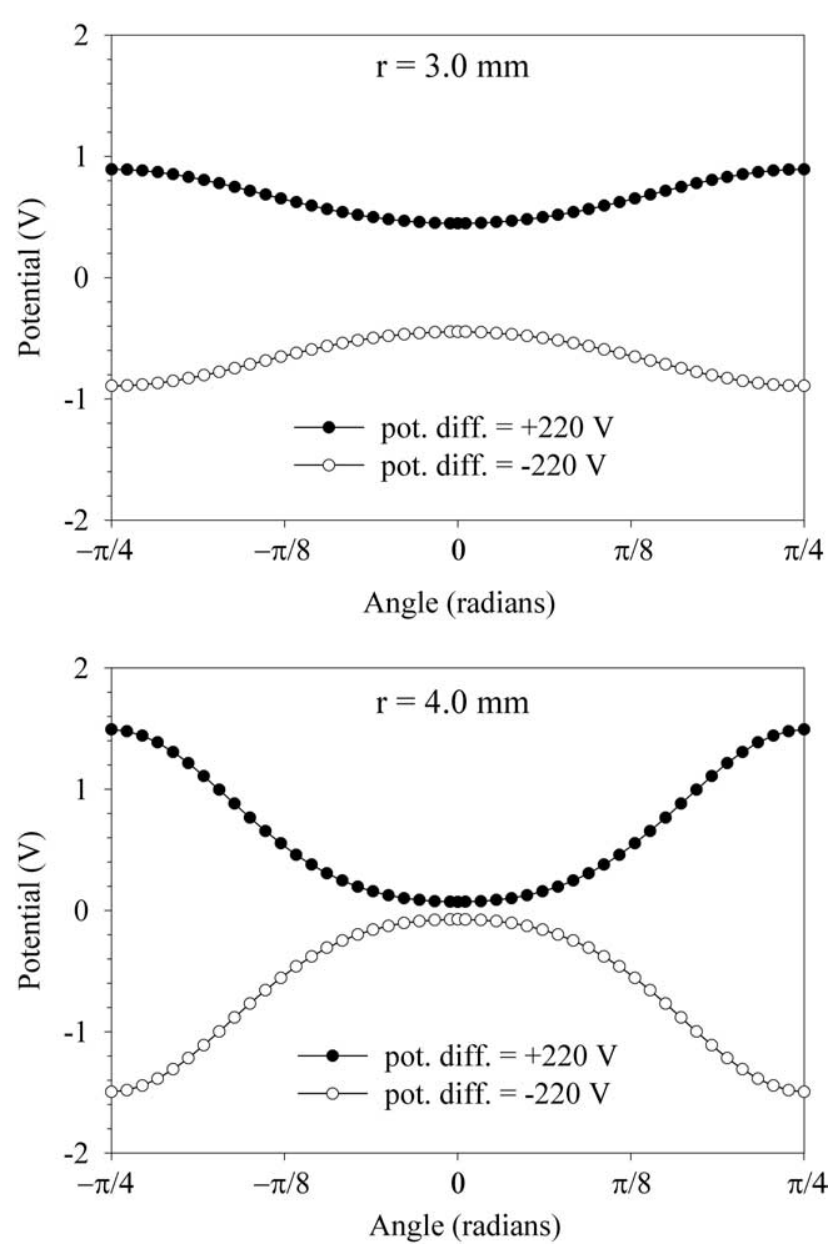

Figure 14. The lower frame shows the dc potential at a radial amplitude of $4.0 \mathrm{~mm}$ for potential differences between the auxiliary electrodes and the quadrupole rod offset of $+220 \mathrm{~V}$ and -220 $\mathrm{V}$ as a function of angle. The upper frame shows the dc potential at a radial amplitude of $3.0 \mathrm{~mm}$. In each case the $\mathrm{rf}$ and $\mathrm{dc}$ potentials applied to the quadrupole rods was $0 \mathrm{~V}$. At the higher radial amplitude the shape of the potential, with $+220 \mathrm{~V}$ applied to the auxiliary electrodes, helps to confine the ions to the region around $\theta=0$. When the applied potential is $-220 \mathrm{~V}$ the shape of the potential causes the ions to move away from the $\theta=0$ region. The effect is less dramatic at lower radial amplitudes (upper frame, $\mathrm{r}=3.0 \mathrm{~mm}$ ) where the shape of the potential is not so distorted as a function of angle.

electrodes changed the shape of the potential well.


point of interest is rotated from $\theta=-\pi / 4$ to $\theta=\pi / 4$. Data is shown for radii of 3 and $4 \mathrm{~mm}$. The quadrupole rod is located at $\theta=0$ radians and the auxiliary electrodes are located at $\theta=-\pi / 4$ and $\theta=\pi / 4$ radians. The static potential produced by $+220 \mathrm{~V}$ applied to the auxiliary electrodes is shown as the solid circles while the static potential produced by $-220 \mathrm{~V}$ is shown as the open circles. The static potential produced by the +220 $\mathrm{V}$ deepens the potential well and helps to confine the motion of the ion parallel to the direction of excitation. When the static potential is negative, the potential well develops a hump at $\theta=0$. Consequently, ions are confined less well to the direction of excitation and may gain radial amplitude perpendicular to the direction of excitation. At lower radial amplitudes, the potential well is affected less strongly and ions are diverted little from the direction of excitation.

\section{Conclusions}

The fragmentation efficiency of a heavy ion, $m / z=2722$ Th, has been increased significantly through the use of a higher order field, specifically a dc octopole. The dc octopole field was created by the addition of four auxiliary electrodes situated between the quadrupole rods at a distance of $10 \mathrm{~mm}$ from the axis of the quadrupole. The content of the dc octopolar field could then be adjusted by varying the potential to the auxiliary electrodes. The inclusion of the dc octopolar field has been shown to cause the ions' frequency of motion to shift out of phase with the excitation signal at high radial amplitudes. This resulted in beat-like trajectories with periods of excitation and de-excitation as the ions' frequency of motion shifted in and out of phase with the auxiliary signal. This led to a reduction in the loss of ions on the quadrupole rods resulting in a high fragmentation efficiency relative to the fragmentation efficiency obtained when using a round rod quadrupole. This process allows the ion to be fragmented more efficiently while maintaining relatively short excitation periods.

\section{Acknowledgments}

The author acknowledges Jim Hager, Yves LeBlanc, Frank Londry, and Michael Guna for the many useful discussions related to this work. The author further acknowledges the critical and entertaining comments made by Frank Londry during his review of this manuscript.

\section{References}

1. Collings, B. A.; Stott, W. R.; Londry, F. A. Resonant Excitation in a Low Pressure Linear Ion Trap. J. Am. Soc. Mass Spectrom. 2003, 14, 622-634.

2. Michaud, A. L.; Frank, A. J.; Ding, C.; Douglas, D. J. Ion Excitation in a Linear Quadrupole Ion Trap with an Added Octopole Field. J. Am. Soc. Mass Spectrom. 2005, 16, 835-849.

3. Hager, J. W. A New Linear Ion Trap Mass Spectrometer. Rapid Commun. Mass Spectrom. 2002, 16, 512-526.

4. Douglas, D. J.; Glebova, T. A.; Konenkov, N. V.; Sudakov, M. Y. Spatial Harmonics of the Field in a Quadrupole Mass Filer with Circular Electrodes. Tech. Phys. 1999, 44, 1215-1219.

5. Sudakov, M.; Douglas, D. J. Linear Quadrupoles with Added Octopole Fields. Rapid Commun. Mass Spectrom. 2003, 17, 2290-2294.

6. Gerlich, D. Inhomogeneous rf Fields: A Versatile Tool for the Study of Processes with Slow Ions. In Advances in Chemical Physics, Vol. 82. Ng, C-Y; Baer, M., eds. John Wiley and Sons, Inc.: New York, 1992, pp 1-176.

7. Franzen, J.; Gabling, R.-H.; Heinen, G.; Weiss, G. Method for Mass Spectroscopic Examination of a Gas Mixture and Mass Spectrometer Intended for Carrying Out This Method. U.S. Patent 5, 028, 777, 1991. 
8. Wang, Y.; Franzen, J.; Wanczek, K. P. The Non-linear Resonance Ion Trap. Part 2. A General Theoretical Analysis. Int. J. Mass Spectrom. Ion Processes 1993, 124, 125-144.

9. Franzen, J. Simulation Study of an Ion Cage with Superimposed Multipole Fields. Int. J. Mass Spectrom. Ion Processes 1991, 106, 63-78.

10. Franzen, J.; Wang, Y. Quadrupole Ion Trap with Switchable Multipole Fractions. U.S. Patent 5, 468, 958, 1995.

11. Franzen, J.; Wang, Y. Quadrupole Ion Trap with Switchable Multipole Fractions. U.S. Patent Re. 36, 906, 1995.

12. Londry, F.; Collings, B. A.; Stott, W. R. Fragmentation of Ions by Resonant Excitation in a Higher Order Multipole Field, Low Pressure Ion Trap. U.S. Patent Application 0189171 A1, 2003.

13. Loboda, A.; Krutchinsky, A.; Loboda, O.; McNabb, J.; Spicer, V.; Ens, W.; Standing, K. Novel LINAC II Electrode Geometry for Creating an Axial Field in a Multipole Ion Guide. Eur. J. Mass Spectrom. 2000, 6, 531-536.
14. Londry, F. A.; Hager, J. W. Mass Selective Axial Ion Ejection from a Linear Quadrupole Ion Trap. J. Am. Soc. Mass Spectrom. 2003, 14, 1130-1147.

15. Flanagan, J. M. Mass Spectrometry Calibration Using Homogeneously Substituted Fluorinated Triazatriphosphorines. U.S. Patent 5,872,357, 1999.

16. Covey, T.; Douglas, D. J. Collision Cross Sections for Protein Ions. J. Am. Soc. Mass Spectrom. 1993, 4, 616-623.

17. Dahl, D. A. Simion 3-D Version 7.0, Publication INEEL-95/0403; Idaho National Engineering and Environmental Laboratory: Idaho Falls, Idaho, 2000.

18. Dehmelt, H. G. Radiofrequency Spectroscopy of Stored Ions: Storage. Adv. Atom. Mol. Phys. 1967, 3, 53-72.

19. Dawson, P. H. Quadrupole Mass Spectrometry and Its Applications; AIP Press: Woodbury, NY, 1995; pp 13-14.

20. French, A. P. Vibrations and Waves; W. W Norton and Company, Inc.: New York, NY, 1971; pp 189-196. 